\title{
VECTOR MEASURES OF BOUNDED SEMIVARIATION AND ASSOCIATED CONVOLUTION OPERATORS
}

\author{
PAULETTE SAAB \\ Department of Mathematics, University of Missouri, Columbia, Missouri 65211, USA \\ email: saabp@missouri.edu \\ and MANGATIANA A. ROBDERA \\ Department of Mathematics, University of Botswana, Gaborone, Botswana \\ email: robdera@yahoo.com
}

(Received 27 January 2010; accepted 9 August 2010; first published online 8 December 2010)

\begin{abstract}
Let $G$ be a compact metrizable abelian group, and let $X$ be a Banach space. We characterize convolution operators associated with a regular Borel $X$-valued measure of bounded semivariation that are compact (resp; weakly compact) from $L^{1}(G)$, the space of integrable functions on $G$ into $L^{1}(G) \check{\otimes} X$, the injective tensor product of $L^{1}(G)$ and $X$. Along the way we prove a Fourier Convergence theorem for vector measures of relatively compact range that are absolutely continuous with respect to the Haar measure.
\end{abstract}

2010 Mathematics Subject Classification. 46G10, 46B99.

1. Notation and preliminaries. Throughout this note we let $G$ be a compact metrizable abelian group, $\widehat{G}$ be its dual group, $\Sigma$ be the $\sigma$-field of Borel subsets of $G$, and we let $m$ denote a normalized Haar measure on $G$. If $X$ is a Banach space, we shall denote by $C(G, X)$ the Banach space of all continuous $X$-valued functions $\varphi$ defined on $G$ equipped with the supremum norm, $\|\varphi\|_{\infty}=\sup _{t \in G}\|\varphi(t)\|$. We denote by $M(G, X)$ the Banach space of all regular Borel $X$-valued measures $\lambda$ defined on $\Sigma$ that are of bounded variation equipped with the variation norm, $\|\lambda\|=|\lambda|(G)=$ $\sup _{\Pi} \sum_{A \in \Pi}\|\lambda(A)\|$, where the supremum is taken over all finite partitions $\Pi$ of $G$ into elements of $\Sigma$. More generally, we let $M_{b}(G, X)$ the Banach of all regular Borel $X$-valued measures $\lambda$ defined on $\Sigma$ equipped with the semivariation norm, \|\|$\lambda\|=\| \lambda \|(G)=\sup _{\left\|x^{*}\right\| \leq 1}\left|x^{*} \lambda\right|(G)$. We also denote by $L^{p}(G, X), 1 \leq p<\infty$, the Banach space of (all equivalence classes of) $m$-Bochner $p$-integrable function $f$ on $G$ with values in $X$, under the norm $\|f\|_{p}=\left(\int_{G}\|f(t)\|^{p} d m(t)\right)^{\frac{1}{p}}$. If $X=\mathbb{R}$ or $\mathbb{C}$, we shall simply write $C(G)$ (resp., $M(G))$ for $C(G, X)$ (resp., $M(G, X))$, and $L^{p}(G)$ for $L^{p}(G, X)$.

If $\mu \in M_{b}(G, X)$, the Fourier Transform of $\mu$, denoted by $\hat{\mu}$, is the $X$-valued function defined on $\widehat{G}$ as follows:

$$
\hat{\mu}(\gamma)=\int_{G} \bar{\gamma}(t) d \mu(t), \text { for each } \gamma \in \widehat{G}
$$

A measure $\mu \in M_{b}(G, X)$ is said to have a relatively compact range if $\mu(\Sigma)=$ $\{\mu(A): A \in \Sigma\}$ is relatively compact in $X$. 
If $\mu \in M_{b}(G, X)$, and $f$ is a Borel function on $G$, then the convolution off and $\mu$, denoted as $f * \mu$, is the $X$-valued function defined on $G$ by

$$
f * \mu(s)=\int_{G} f(s-t) d \mu(t)
$$

for each $s$ in $G$, provided that the integral exists.

The next proposition shows that the integral in $\left(^{*}\right)$ is well defined for elements in $C(G)$, and that the notion of convolution can be extended to elements in $L^{1}(G)$. In what follows, $L^{1}(G) \otimes x X$ will stand for the injective tensor product of $L^{1}(G)$ and the Banach space $X$. We recall that $L^{1}(G) \ddot{\otimes} X$ is the completion of $L^{1}(G) \otimes X$ under the injective tensor norm \|\|$_{\vee}$ defined as follows: if $u=\sum_{i=1}^{n} f_{i} \otimes x_{i} \in L^{1}(G) \otimes X$

$$
\begin{aligned}
\|u\|_{\vee} & =\sup \left\{\left|\sum_{i=1}^{n} y^{*}\left(f_{i}\right) x^{*}\left(x_{i}\right)\right|:\left\|y^{*}\right\| \leq 1,\left\|x^{*}\right\| \leq 1, y^{*} \in\left(L^{1}(G)\right)^{*}, x^{*} \in X^{*}\right\} \\
& =\sup \left\{\left\|\sum_{i=1}^{n} x^{*}\left(x_{i}\right) f_{i}\right\|_{1}:\left\|x^{*}\right\| \leq 1, x^{*} \in X^{*}\right\} . \\
& =\sup \left\{\left\|x^{*} u\right\|_{1}:\left\|x^{*}\right\| \leq 1, x^{*} \in X^{*}\right\} .
\end{aligned}
$$

Proposition 1.1. Let $X$ be a Banach space, $G$ be a compact metrizable abelian group, and let $\mu \in M_{b}(G, X)$.

If $f \in C(G)$, then $f * \mu \in C(G, X)$ with

$$
\|f * \mu\|_{\infty} \leq\|f\|_{\infty}\|\mu\|(G) .
$$

Moreover,

$$
\|f * \mu\|_{\vee} \leq\|f\|_{1}\|\mu\|(G)
$$

Proof. Let $d$ denote the metric defining the topology of $G$. Let $f \in C(G)$, since $f$ is uniformly continuous on $G$, for each $\epsilon>0$, there exists $\delta>0$, such that

$$
d\left(u_{1}, u_{2}\right)<\delta \Longrightarrow\left|f\left(u_{2}\right)-f\left(u_{1}\right)\right|<\epsilon .
$$

If $d\left(s_{1}, s_{2}\right)<\delta$, then

$$
\begin{aligned}
& \left\|f * \mu\left(s_{2}\right)-f * \mu\left(s_{1}\right)\right\|=\sup _{\left\|x^{*}\right\| \leq 1}\left|\int_{G} f\left(s_{2}-t\right)-f\left(s_{1}-t\right) d x^{*} \mu(t)\right| \\
& \leq \sup _{\left\|x^{*}\right\| \leq 1} \int_{G}\left|f\left(s_{2}-t\right)-f\left(s_{1}-t\right)\right| d\left|x^{*} \mu\right|(t) \leq \epsilon\|\mu\|(G) .
\end{aligned}
$$

This shows that $f * \mu$ is uniformly continuous on $G$, and (**) follows easily.

To prove $\left({ }^{* *}\right)$, let $f \in C(G)$, then for each $x^{*} \in X^{*}$, it is easy to check that for each $s \in G$,

$$
x^{*}(f * \mu(s))=x^{*}\left(\int_{G} f(s-t) d \mu(t)\right)
$$




$$
=\int_{G} f(s-t) d x^{*} \mu(t)=f * x^{*} \mu(s) .
$$

Thus for each $f \in C(G)$ and $x^{*} \in X^{*}$, we have

$$
x^{*}(f * \mu)=f * x^{*} \mu,
$$

where $f * x^{*} \mu$ is the convolution of $f$ and the scalar measure $x^{*} \mu$. In particular, since $f * x^{*} \mu \in L^{1}(G)$, it follows from convolutions with regular Borel scalar measures (see [2] or [5]) that the norm in $L^{1}(G)$ of $f * x^{*} \mu$ satisfies

$$
\left\|f * x^{*} \mu\right\|_{1} \leq\|f\|_{1}\left|x^{*} \mu\right|(G)
$$

hence,

$$
\|f * \mu\|_{\vee}=\sup _{\left\|x^{*}\right\| \leq 1}\left\|f * x^{*} \mu\right\|_{1} \leq\|f\|_{1}\|\mu\|(G)
$$

It follows from $(* *)$ that each element $\mu$ in $M_{b}(G, X)$ induces a bounded linear operator

$$
T_{\mu}: C(G) \rightarrow C(G, X)
$$

where

$$
T_{\mu} f=f * \mu \quad \text { for each } f \in C(G)
$$

Since $C(G)$ is dense in $L^{1}(G)$, it follows from (***) that the notion of convolution with a vector measure $\mu$ can be extended to elements in $L^{1}(G)$, and that $T_{\mu}$ extends to a bounded linear operator

$$
T_{\mu}: L^{1}(G) \rightarrow L^{1}(G) \check{\otimes} X,
$$

where for each $f \in L^{1}(G), T_{\mu}(f)$ will also be denoted by $f * \mu$.

The operator $T_{\mu}$ is called a convolution operator associated to the regular Borel vector measure $\mu$. Such an operator was studied in [4], under the additional assumption that the measure $\mu$ is of bounded variation, in which case $T_{\mu}$ is actually a bounded linear operator from $L^{1}(G)$ into $L^{1}(G, X)$.

The work in [4] was motivated by the following result, where compact and weakly compact convolution operators on $C(G)$ and $L^{1}(G)$ were completely characterized when the underlying measure is a regular Borel scalar measure.

THEOREM 1.2. Let $G$ be a compact metrizable abelian group with Haar measure $m$, and let $v \in M(G)$. The following assertions about convolution operators are equivalent:

1. $T_{v}: L^{1}(G) \mapsto L^{1}(G)$ is compact.

2. $T_{v}: L^{1}(G) \mapsto L^{1}(G)$ is weakly compact.

3. $T_{v}: C(G) \mapsto C(G)$ is compact.

4. $T_{v}: C(G) \mapsto C(G)$ is weakly compact.

5. the measure $v$ is absolutely continuous with respect to Haar measure $m$. 
When the regular Borel $X$-valued vector measure $\mu$ is assumed to be of bounded variation, the results of [4] can be summarized in the following theorems.

THEOREM 1.3. Let $G$ be a compact metrizable abelian group with Haar measure $m$, let $X$ be a Banach space and let $\mu \in M(G, X)$. The following assertions about convolution operators are equivalent:

(i) $T_{\mu}: L^{1}(G) \mapsto L^{1}(G, X)$ is compact.

(ii) $T_{\mu}: L^{1}(G) \mapsto L^{1}(G, X)$ is weakly compact.

(iii) The measure $\mu=\phi . m$, where $\phi \in L^{1}(G, X)$.

THEOREM 1.4. Let $G$ be a compact metrizable abelian group with Haar measure $m$, let $X$ be a Banach space and let $\mu \in M(G, X)$. Then

(i) $T_{\mu}: C(G) \mapsto C(G, X)$ is weakly compact if and only if the measure $\mu$ is absolutely continuous with respect to Haar measure.

(ii) $T_{\mu}: C(G) \mapsto C(G, X)$ is compact if and only if the measure $\mu$ is absolutely continuous with respect to Haar measure, and has relatively compact range.

As one can see, when the measure $\mu \in M(G, X)$, the assertion ' $T_{\mu}: L^{1}(G) \mapsto$ $L^{1}(G, X)$ is compact' implies ' $T_{\mu}: C(G) \mapsto C(G, X)$ is compact', but the reverse implication is no longer true. One object of this note is to show that when $\mu \in M_{b}(G, X)$, then $T_{\mu}: L^{1}(G) \mapsto L^{1}(G) \check{\otimes} X$ is compact if and only if $T_{\mu}: C(G) \mapsto C(G, X)$ is compact.

A key ingredient that is very useful in dealing with convolution operators is the notion of an approximate unit also referred to as a summability kernel. Recall that a trigonometric polynomial $p$ is a continuous function on the group $G$ of the form $p=\sum_{i=1}^{n} \alpha_{i} \gamma_{i}$, where for each $1 \leq i \leq n, \alpha_{i}$ is a scalar, and $\gamma_{i} \in \widehat{G}$. When the compact group $G$ is metrizable it is possible to find a sequence of trigonometric polynomials whose main properties are outlined in the following proposition (see [2]).

Proposition 1.5. Let $G$ be a compact metrizable abelian group. There exists a sequence $\left(k_{n}\right)$ of trigonometric polynomials such that:

1. $k_{n} \geq 0$ for each $n \in \mathbb{N}$.

2. $\left\|k_{n}\right\|_{1}=1$.

3. $\lim _{n} \int_{V^{c}} k_{n}(t) d m(t)=0$, for each neighbourhood $V$ of zero in $G$.

In the sequel we will refer to the sequence $\left(k_{n}\right)$ as a an approximate unit or $a$ summability kernel associated with the group $G$.

Another important property (see [2]) of an approximate unit that will be useful in the sequel is the following:

Proposition 1.6. Let $G$ be a compact metrizable abelian group, $\phi$ a continuous function on $G$ and $\left(k_{n}\right)$ an approximate unit. Then

$$
\lim _{n \rightarrow \infty} \int_{G} k_{n}(t) \phi(t) d m(t)=\phi(0),
$$

where 0 denotes the identity element of the group $G$.

Finally, all notions not defined can be found in $[\mathbf{1}, \mathbf{2}, \mathbf{3}, \mathbf{5}]$ or $[\mathbf{6}]$. 
2. The main theorem. A very important and widely used result in classical Fourier analysis is the Fourier Convergence Theorem in which approximate units are used to approximate elements of $C(G)$ and $L^{1}(G)$. A straight forward modification of the proof in the scalar case (see [2]) enables us to generalize these facts to vector-valued functions. Namely, it can be shown that if $f \in L^{1}(G, X)$ (resp. $\left.C(G, X)\right)$ and $\left(k_{n}\right)$ is an approximate unit, then

$$
\begin{gathered}
\left\|k_{n} * f-f\right\|_{1} \longrightarrow 0 \text { as } n \longrightarrow \infty, \\
\text { (resp. } \left.\left\|k_{n} * f-f\right\|_{\infty} \longrightarrow 0 \text { as } n \longrightarrow \infty\right) .
\end{gathered}
$$

One result that we shall show in this paper is an extension of the Fourier Convergence Theorem to vector measures of bounded semivariation, that are absolutely continuous with respect to Haar measure and that have a compact range. In what follows we shall denote by $\mathcal{K}(G, X)$ the subspace of $M_{b}(G, X)$ of such measures. It is a well-known fact (see [1]) that $\mathcal{K}(G, X)$ is a Banach space when endowed with the semivariation norm and that it is isometrically isomorphic to $L^{1}(G) \check{\otimes} X$.

THEOREM 2.1. Let $G$ be a compact metrizable abelian group with Haar measure $m$, and let $X$ be a Banach space. The following assertions about a regular Borel $X$-valued measure of bounded semivariation $\mu$ are equivalent:

(i) $\mu \in \mathcal{K}(G, X)$.

(ii) For any approximate unit $\left(k_{n}\right)$, $\left\|\left|k_{n} * \mu-\mu\right|\right\| \longrightarrow 0$ as $n \longrightarrow \infty$.

Proof. To prove (i) $\Rightarrow$ (ii) assume that $\mu \ll m$ and that the measure $\mu$ has a relatively compact range. Let $S: C(G) \mapsto X$ be the operator

$$
S(f)=\int_{G} f d \mu \text { for each } f \in C(G) .
$$

Since $\mu$ has relatively compact range, it follows (see [1]) that the operator $S$ is compact. Moreover, it is easy to check that for each $x^{*} \in X^{*}$,

$$
S^{*}\left(x^{*}\right)=x^{*} \mu
$$

This shows that when $\mu \ll m$ and $\mu$ has relatively compact range the set $\left\{x^{*} \mu\right.$ : $\left.\left\|x^{*}\right\| \leq 1\right\}$ is a compact subset of $L^{1}(G)$. In particular, this implies that

$$
\left\|k_{n} * x^{*} \mu-x^{*} \mu\right\|_{1} \longrightarrow 0 \text { uniformly in }\left\|x^{*}\right\| \leq 1 .
$$

This of course is equivalent to

$$
\left\|k_{n} * \mu-\mu\right\| \mid \longrightarrow 0 .
$$

The implication (ii) $\Rightarrow$ (i) follows from the fact that for each $n \in \mathbb{N}$,

$$
k_{n} * \mu \in L^{1}(G) \check{\otimes} X
$$

and the fact that $\mathcal{K}(G, X)$ is isometrically isomorphic to $L^{1}(G) \check{\otimes} X$.

We are now ready to state the main result of this paper. 
THEOREM 2.2. Let $G$ be a compact metrizable abelian group with Haar measure $m$. Let $X$ be a Banach space and let $\mu$ be an $X$-valued measure of bounded semivariation. The following assertions about the convolution operator $T_{\mu}$ are equivalent:

(a) $T_{\mu}: L^{1}(G) \mapsto L^{1}(G) \ddot{\otimes} X$ is compact.

(b) $T_{\mu}: L^{1}(G) \mapsto L^{1}(G) \check{\otimes} X$ is weakly compact.

(c) $\mu$ is absolutely continuous with respect to $m$ and the measure $\mu$ has a relatively compact range.

(d) $T_{\mu}: C(G) \mapsto C(G, X)$ is compact.

Proof. (a) $\Rightarrow$ (b) is obvious. To prove (b) $\Rightarrow$ (c) note that if $T_{\mu}: L^{1}(G) \mapsto L^{1}(G) \check{\otimes} X$ is weakly compact, then if $\left(k_{n}\right)$ is an approximate unit the set

$$
\left\{k_{n} * \mu, n \in \mathbb{N}\right\}
$$

is weakly compact in $L^{1}(G) \ddot{\otimes} X$. Thus, there exit a subsequence $\left(k_{n_{j}}\right)$ of $\left(k_{n}\right)$ and an element $v \in L^{1}(G) \otimes \check{\otimes} X$, such that

$$
k_{n_{j}} * \mu \longrightarrow v \text { weakly in } L^{1}(G) \check{\otimes} X .
$$

In particular, for each $\gamma \in \widehat{G}$,

$$
\widehat{k_{n_{j}} * \mu}(\gamma) \longrightarrow \hat{v}(\gamma) \text { weakly in } X
$$

But it is easy to check that for each $\gamma \in \widehat{G}$,

$$
\widehat{k_{n_{j}} * \mu}(\gamma)=\widehat{k_{n_{j}}}(\gamma) \hat{\mu}(\gamma)
$$

and by Proposition 1.6

$$
\widehat{k_{n}}(\gamma) \longrightarrow 1
$$

it follows that for each $x^{*} \in X^{*}$,

$$
x^{*}(\hat{\mu}(\gamma))=x^{*}(\hat{v}(\gamma)) .
$$

By the Uniqueness theorem (see [5]) we have that for $x^{*} \in X^{*}$, the scalar measures

$$
x^{*} \mu=x^{*} v
$$

and hence by the Hahn-Banach Theorem, $\mu=v$ and the measure $\mu$ has a relatively compact range.

Moreover, when $T_{\mu}: L^{1}(G) \mapsto L^{1}(G) \check{\otimes} X$ is weakly compact, then for each $x^{*} \in$ $X^{*}$, the bounded linear operator from $L^{1}(G)$ into $L^{1}(G)$, which to each $f \in L^{1}(G)$, associates the element $x^{*}(f * \mu)$ is also weakly compact. But it is easy to check that for each $f \in L^{1}(G)$, and $x^{*} \in X^{*}$

$$
x^{*}(f * \mu)=f * x^{*} \mu .
$$

Thus, the convolution operator $T_{x^{*} \mu}: L^{1}(G) \mapsto L^{1}(G)$ is also weakly compact, and therefore $x^{*} \mu \ll m$ for each $x^{*} \in X^{*}$ by Theorem 1.2. It follows that $\mu(A)=0$ whenever $A$ is a Borel subset of $G$ and $m(A)=0$. Since the measures $\mu$ and $m$ are countably 
additive on the $\sigma$-field $\Sigma$ of Borel subsets of $G$, it follows from a result of B. J. Pettis (see [1]) that $\mu \ll m$.

To prove (c) $\Rightarrow$ (a) assume that $\mu \ll m$ and that the measure $\mu$ has a relatively compact range. By Theorem 2.1

$$
\left\|k_{n} * \mu-\mu\right\| \mathrm{I} 0 .
$$

To finish the proof note that by Proposition 1.1

$$
\left\|T_{\mu}-T_{k_{n} * \mu}\right\| \leq\left\|\mid k_{n} * \mu-\mu\right\| \| .
$$

Moreover, for each $n \in \mathbb{N}$, we have

$$
T_{k_{n} * \mu}=T_{k_{n}} \circ T \mu .
$$

By Theorem 1.2 the operator $T_{k_{n}}$ is compact for each $n \in \mathbb{N}$, and consequently each operator $T_{k_{n} * \mu}$ is compact. This of course implies that $T_{\mu}$ is compact.

The proof of (d) $\Rightarrow$ (c) follows from the fact that when $T \mu: C(G) \mapsto C(G, X)$ is compact, then for each $x^{*} \in X^{*}$, the convolution operator $T_{x^{*} \mu}: C(G) \mapsto C(G)$ is also compact, and therefore $x^{*} \mu \ll m$ for each $x^{*} \in X^{*}$ by Theorem 1.2. This of course shows that $\mu \ll m$.

Moreover, one can proceed as in [4] to show that for each Borel subset $A$ of $G$,

$$
T_{\mu}^{* *}\left(1_{A}\right)=1_{A} * \mu .
$$

Because $T_{\mu}$ is compact, its second adjoint $T_{\mu}^{* *}$ is compact, and it takes its values in $C(G, X)$. Therefore, the set $\left\{1_{A} * \mu: A \in \Sigma\right\}$ is relatively compact in $C(G, X)$, and therefore the set $\mu(\Sigma)=\left\{1_{-A} * \mu(0): A \in \Sigma\right\}$ is relatively compact in $X$.

To prove (c) $\Rightarrow$ (d) assume that $\mu \ll m$, that the measure $\mu$ has a relatively compact range, and consider the set $\mathcal{F}=\left\{f * \mu: f \in C(G),\|f\|_{\infty} \leq 1\right\}$. Since $\mu$ has relatively compact range, then for each $s \in G$, if we denote by $\mu_{s}=\sigma_{s}(\mu)$, the measure image of $\mu$ by the translation $\sigma_{s}: G \mapsto G$ such that $\sigma_{s}(t)=s-t$, then $\mu_{s}=\sigma_{s}(\mu)$ has also a relatively compact range. Thus for each $s \in G$, the set

$$
\begin{aligned}
\mathcal{F} & (s)=\left\{f * \mu(s): f \in C(G),\|f\|_{\infty} \leq 1\right\} \\
& =\left\{\int_{G} f(s-t) d(\mu)(t): f \in C(G),\|f\|_{\infty} \leq 1\right\} \\
& =\left\{\int_{G} f(t) d \sigma_{s}(\mu)(t): f \in C(G),\|f\|_{\infty} \leq 1\right\}
\end{aligned}
$$

is relatively compact in $X$.

To finish the proof we will be done if we could show that the set $\mathcal{F}$ is equicontinuous. To this end note that for $s, s^{\prime} \in G$ and $\|f\|_{\infty} \leq 1$ in $C(G)$,

$$
\begin{array}{rl}
\| f & * \mu(s)-f * \mu\left(s^{\prime}\right)\|=\| \int_{G} f(s-t)-f\left(s^{\prime}-t\right) d \mu(t) \| \\
& =\sup _{\left\|x^{*}\right\| \leq 1}\left|\int_{G} f(s-t)-f\left(s^{\prime}-t\right) d x^{*} \mu(t)\right| \\
& =\sup _{\left\|x^{*}\right\| \leq 1}\left|\int_{G} f(t) d x^{*}\left(\mu_{s}-\mu_{s^{\prime}}\right)(t)\right|
\end{array}
$$




$$
\begin{aligned}
& \leq \sup _{\left\|x^{*}\right\| \leq 1} \int_{G}|f(t)| d\left|\left(x^{*} \mu_{s}-x^{*} \mu_{s^{\prime}}\right)\right|(t) \\
& \leq\left\|\mu_{s}-\mu_{s^{\prime}}\right\|(G) .
\end{aligned}
$$

Since the set $\left\{x^{*} \mu:\left\|x^{*}\right\| \leq 1\right\}$ is compact in $L^{1}(G)$, and since for each $g \in L^{1}(G)$ the map $s \mapsto g_{s}$ is continuous from $G$ to $L^{1}(G)$ (see [5]), it follows that the map $s \mapsto$ $x^{*} \mu_{s}$ is uniformly equicontinuous in $x^{*} \in X^{*},\left\|x^{*}\right\| \leq 1$. This implies that the map $s \mapsto \mu_{s}$ is uniformly continuous from $G$ into $M_{b}(G, X)$, and shows that the set $\mathcal{F}$ is equicontinuous and thus by Ascoli's Theorem the set $\mathcal{F}$ is relatively compact in $C(G, X)$.

\section{REFERENCES}

1. J. Diestel and J. J. Uhl, Jr., Vector Measures, Amer. Math. Soc. Surveys, Vol. 15, (Providence, Rhode Island, 1977).

2. $\mathrm{D} . \mathrm{Li}$ and $\mathrm{H}$. Quefélec, Introduction à l'étude des espaces de Banach, Société Mathématique (de France, France, 2004).

3. J. Lindenstrauss and L. Tzafriri, Classical Banach Spaces I (Springer-Verlag, Berlin and New York, 1977).

4. M. A. Robdera and P. Saab, Convolution operators associated with vector measures, Glasgow Math. J. 40 (1998), 367-384.

5. W. Rudin, Fourier analysis on groups, in Interscience Tracts in Pure and Applied Mathematics, No. 12 (Interscience, New York, 1962).

6. P. Wojtaszcyk, Banach spaces for analysts (Cambridge University Press, 1991). 